SHORT REPORT

\title{
Multiple pathologies in a patient with a progressive neurodegenerative syndrome
}

\author{
T-W Liang, M S Forman, J E Duda, L McCluskey, J Q Trojanowski, A Siderowf
}

J Neurol Neurosurg Psychiatry 2005;76:252-255. doi: 10.1136/jnnp.2004.039479

A woman presenting with levodopa responsive Parkinsonism developed rapidly progressive bulbar signs, quadriparesis, and upper and lower motor neurone signs. At necropsy, she was found to have three pathological diagnoses: amyotrophic lateral sclerosis, Parkinson's disease, and abundant tau-positive argyrophilic neuritic pathology, known as argyrophilic grain disease. This case raises the possibility that three distinct neuropathological diagnoses share a common aetiology.

$\mathrm{P}$ arkinson's disease, amyotrophic lateral sclerosis (ALS), and argyrophilic grain disease (AGD) are generally viewed as distinct clinicopathological entities. Outside specific geographical regions such as the island of $\mathrm{Guam}^{1}$ and the Kii peninsula of $\mathrm{Japan}^{2}$ the combination of ALS and Parkinson's disease is highly unusual. Furthermore, western Pacific parkinsonism-dementia complex/ALS (PDC-ALS) is pathologically distinct from common Parkinson's disease and ALS because of the abundance of Alzheimer-type neurofibrillary tangles. ${ }^{1}$ In the western world, the rare cases of ALS with nigral degeneration that have been reported are referred to as ALS-plus. ${ }^{3}$ Combinations of neurodegenerative disorders reported include progressive supranuclear palsy (PSP) and Parkinson's disease, ${ }^{4}$ PSP and multiple system atrophy (MSA), ${ }^{5}$ ALS and the cerebellar form of MSA, ${ }^{6}$ and the combination of Alzheimer's disease, Parkinson's disease, ALS, and diffuse Lewy bodies in a single patient. ${ }^{7}$ Bearing in mind that many such cases go unreported, it is tempting to believe that the co-occurrence of multiple neurodegenerative disorders is more frequent than would be expected by chance.

We report the case of a patient who presented with clinical features of both Parkinson's disease and amyotrophy. At necropsy, the patient was found to have the hallmark pathological features of three distinct neurodegenerative disorders marked by tau, $\alpha$-synuclein, and ubiquitin inclusions. We will review the clinical and pathological features of her case and briefly discuss the common underlying mechanisms of inclusion formation and protein aggregation.

\section{CASE HISTORY}

A 61 year old Irish American woman presented with a two year history of rest tremor affecting the right leg. Previous medical history was unremarkable. Family history was notable for a maternal grandmother with Parkinson's disease. She was diagnosed with Parkinson's disease and treated initially with selegiline. Levodopa was added six months later with good response. Three years later, a dopamine agonist was added to control motor fluctuations.

She was first seen at our centre five years after diagnosis for evaluation of Parkinson's disease with motor complications. On examination, she had mild hypophonic dysarthria, facial masking, and moderate parkinsonism with preserved postural reflexes and moderate dyskinesias. No pyramidal signs or cognitive impairment were noted.

Over the next six months, she developed progressive dysarthria, dysphonia, and dysphagia requiring gastrostomy. She also developed difficulty with balance and a waning benefit from dopaminergic drug treatment. Follow up examination showed slowed tongue movements without fasciculations and a combination of spasticity, bilateral Babinski signs, and generalised muscle atrophy. Though she required an augmentative communication device, she had no evidence of cognitive impairment. Electromyography and nerve conduction studies showed no evidence of active or ongoing denervation. Brain magnetic resonance imaging showed scattered non-specific small foci of white matter hyperintensity.

Over the course of the next year, she became bed-bound, rigid, and quadriparetic. Fasciculations were noted in both arms. In the final year of her life, she was unable to communicate except through voluntary eye movements, but did not appear demented. She died from cardiopulmonary arrest eight years after the onset of her initial symptoms.

\section{Pathology}

Gross neuropathological examination revealed a symmetrical brain without significant atrophy (weight $1332 \mathrm{~g}$ ). Horizontal sections through the brain stem showed moderate depigmentation of the substantia nigra and locus coeruleus (fig lA). In addition, the ventral roots of the spinal cord were moderately atrophic.

Microscopic examination revealed an unusual constellation of findings (table 1). In the spinal cord, there was loss of motor neurones in the anterior horn, brain stem, and motor cortex (fig 1D) associated with long tract degeneration and myelin loss in both the lateral and anterior corticospinal tracts (fig 1B). Many surviving motor neurones contained eosinophilic inclusions (fig $1 G$ ) which were reactive to antiubiquitin antibodies (fig $1 \mathrm{H}$ ) but non-reactive to tau and $\alpha$-synuclein, peripherin, $\alpha \beta$-crystallin, and $\alpha$-internexin antibodies. Immunohistochemistry with a panel of antibodies to low, medium, and high molecular weight neurofilament proteins revealed abundant spheroids (data not shown).

In the substantia nigra and locus coeruleus there was profound neurone loss with gliosis and occasional eosinophilic hyalinised inclusions. Immunohistochemistry with antibodies to $\alpha$-synuclein revealed abundant Lewy bodies and neurites throughout the brain stem including the substantia nigra, locus coeruleus, and dorsal motor nucleus of the vagus (fig $1 F$ ). Lewy bodies were also identified in the striatum and limbic structures, notably the amygdala and the cingulate gyrus.

Abbreviations: AGD, argyrophilic grain disease; ALS, amyotrophic lateral sclerosis; DLB, dementia with Lewy bodies; MSA, multiple system atrophy; PSP, progressive supranuclear palsy 

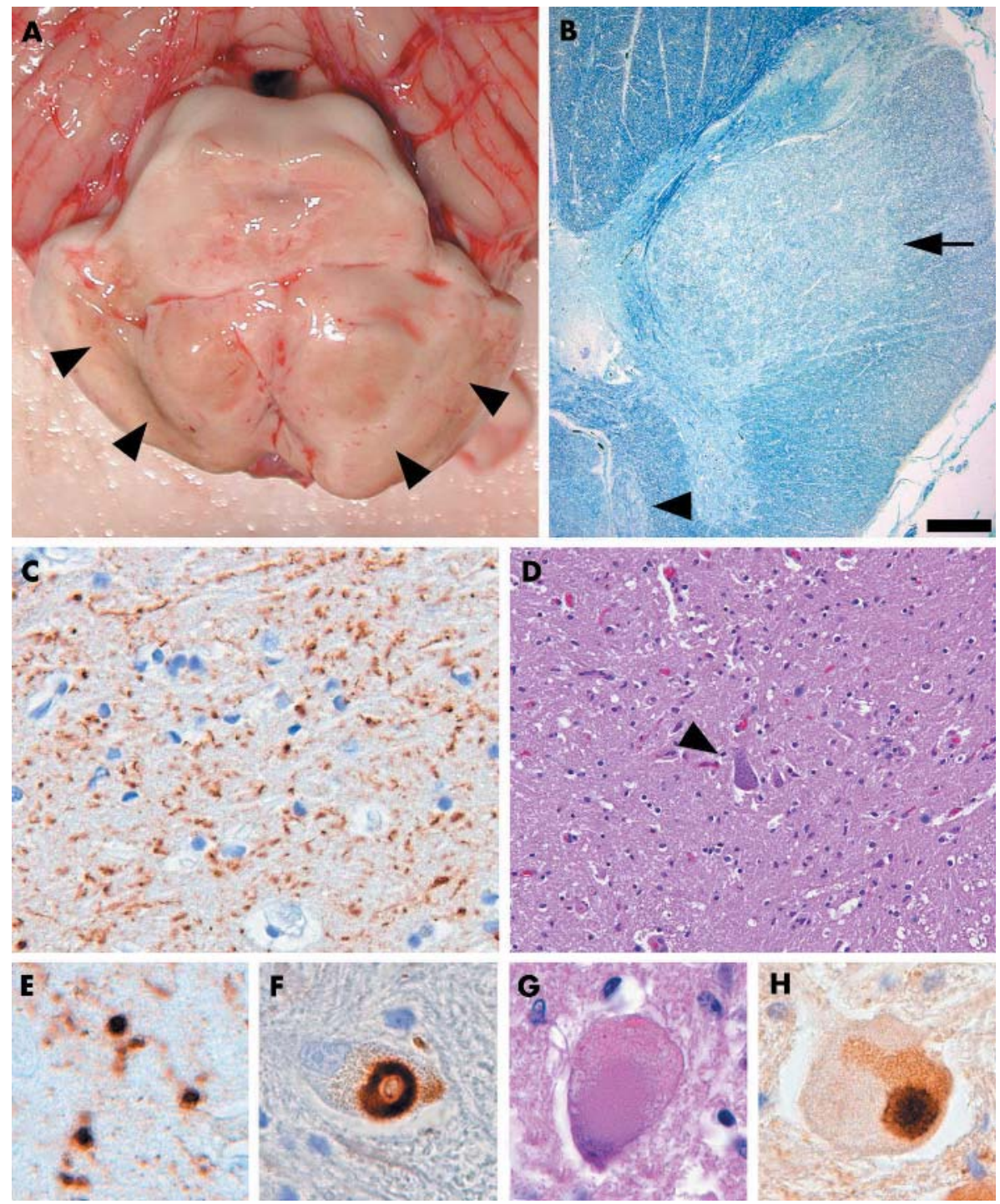

Figure 1 Neuropathology showing an unusual constellation of findings including amyotrophic lateral sclerosis, Parkinson's disease, and argyrophilic grain disease. (A) Gross photograph of midbrain showing marked depigmentation of substantia nigra (arrowheads). (B) Cross section of thoracic spinal cord with degeneration of long tracts including lateral corticospinal tract (arrow) and anterior corticospinal tract (arrowhead) (Kluver Barrera stain). (C) Argyrophilic grains in CA1 region of the hippocampus (anti-phosphorylated tau, PHF1). ${ }^{8}$ (D) Loss of large motor neurones from the anterior horn of the cervical spinal cord (haematoxylin and eosin). The single residual motor neurone is indicated (arrowhead). (E) Grains in the CA1 region of the hippocampus (anti-phosphorylated tau, AT10). (F) $\alpha$-Synuclein positive Lewy body in residual neurone of the substantia nigra (anti- $\alpha$-synuclein, Syn303). ${ }^{9}$ (G) Pale eosinophilic hyaline inclusion in residual motor neurone of anterior horn (haematoxylin and eosin stain). (H) Ubiquitinated spherical cytoplasmic inclusion in surviving motor neurone of anterior horn (anti-ubiquitin 1510, Chemicon International). Scale bar, $500 \mu \mathrm{m}$ (B), $25 \mu \mathrm{m}$ (C), 50 $\mu \mathrm{m}(\mathrm{D}), 12.5 \mu \mathrm{m}(\mathrm{E}-\mathrm{H})$.

In addition, there was abundant tau-positive argyrophilic grain pathology throughout the limbic system including the hippocampal formation, entorhinal cortex, and amygdala (fig 1, C and E). Consistent with published reports, the grain pathology stained robustly with Gallyas silver stain and tau antibodies, but only a small fraction of the pathology was detected with thioflavin S staining. ${ }^{8}$ As in previous reports, argyrophilic grains were immunoreactive with a panel of phosphorylation dependent tau antibodies including AT270 (pThr181), AT8 (pSer202/Thr205), AT10 (pThr212/Ser214) (fig 1E), PHF6 (pThr231), and PHFl (pSer396/Ser404) (fig lC), while non-reactive with 12E8 (pSer262). ${ }^{10}$ There was only mild neuronal and glial tau pathology throughout the remainder of the neocortex, basal ganglia, and brain stem (Braak grade II). Lastly, neither neuritic plaques nor diffuse plaques were detected with either thioflavin S staining or $\beta$ amyloid immunohistochemistry.

\section{DISCUSSION}

The clinical history reflects the two distinct entities of levodopa responsive parkinsonism and amyotrophy. Differentiating this case from cases of ALS-plus is the fact that parkinsonism associated with this group of disorders is not typically responsive to levodopa. ${ }^{3}$ After developing features atypical for Parkinson's disease, additional diagnostic considerations included MSA and concurrent ALS. Necropsy examination showed pathological evidence of Parkinson's disease, ALS, and AGD, explaining the complexity of the clinical presentation. Detailed immunohistochemical analysis further confirmed our diagnoses.

AGD was initially described as a novel neuropathological entity in 1987 in a necropsy series of dementia cases. ${ }^{11}$ Argyrophilic grains were so named because of their minute spindle and comma shaped morphology and the characteristic recognition by silver staining techniques, such as 
Table 1 Distribution of pathological lesions

\begin{tabular}{|c|c|c|c|}
\hline & $\begin{array}{l}\alpha \text {-Synuclein } \\
\text { pathology }\end{array}$ & $\begin{array}{l}\text { Tau } \\
\text { pathology }\end{array}$ & $\begin{array}{l}\text { Ubiquitin } \\
\text { pathology }\end{array}$ \\
\hline Neocortex & 0 & + & 0 \\
\hline Cingulate gyrus & ++ & + & 0 \\
\hline Entorhinal cortex & 0 & +++ & 0 \\
\hline Hippocampus & 0 & +++ & 0 \\
\hline Amygdala & ++ & +++ & 0 \\
\hline Striatum & ++ & + & 0 \\
\hline Globus pallidus & 0 & 0 & 0 \\
\hline Thalamus & 0 & 0 & 0 \\
\hline Subthalamus & 0 & 0 & 0 \\
\hline \multicolumn{4}{|l|}{ Midbrain (except substantia } \\
\hline nigra) & + & + & 0 \\
\hline Substantia nigra & ++ & + & 0 \\
\hline Pons (except locus coeruleus) & + & + & 0 \\
\hline Locus coeruleus & ++ & + & 0 \\
\hline Medulla (except DMN) & + & - & $+(\mathrm{HN})$ \\
\hline Dorsal motor nucleus & ++ & + & 0 \\
\hline Cerebellum & 0 & 0 & 0 \\
\hline Spinal cord, anterior horn & 0 & 0 & ++ \\
\hline \multicolumn{4}{|c|}{$\begin{array}{l}\alpha \text {-Synuclein pathology includes Lewy bodies and Lewy neurites detectec } \\
\text { with Syn } 303 \text {; tau pathology detected with PHF1; ubiquitin pathology } \\
\text { refers to ubiquitin positive, tau, and } \alpha \text {-synuclein negative inclusions } \\
\text { detected with } 1510 \text {. } \\
0 \text {, absent; +, low density; }++ \text {, moderate density; }+++ \text {, high density. } \\
\text { DMN, dorsal nucleus of the vagus; HN, hypoglossal nucleus. }\end{array}$} \\
\hline
\end{tabular}

Bielchowsky, Gallyas, and Bodian preparations. ${ }^{11}$ Immunohistochemistry has revealed that they consist of pathological tau aggregates, relating the disorder to Alzheimer's disease, Pick's disease, corticobasal ganglionic degeneration, and progressive supranuclear palsy.

Biochemically, AGD shows strong similarities to corticobasal ganglionic degeneration and progressive supranuclear palsy. As in other tauopathies, the aggregated tau protein is heavily phosphorylated at multiple serine and threonine residues. ${ }^{10}{ }^{11}$ In the central nervous system six tau isoforms are produced by alternative splicing of exons 2 and 3 in the amino-terminus of the molecule and exon 10 in the carboxyterminus. ${ }^{12}$ The alternative splicing of exon 10 results in either three or four microtubule binding motifs (3R-tau and 4R-tau, respectively). Similar to progressive supranuclear palsy and corticobasal ganglionic degeneration, western blots of insoluble tau fractions consistently show two distinct phospho-tau bands at 64 and $69 \mathrm{kDa}$ and one minor band at $74 \mathrm{kDa}$, all detected by antibodies to the second microtubule binding repeat (that is, 4R-tau). ${ }^{13}$ Upon dephosphorylation, insoluble tau co-migrates with $4 \mathrm{R}$-tau isoforms. ${ }^{14}$ This contrasts with the typical pattern seen in Alzheimer's disease and Pick's disease, in which insoluble tau is composed of approximately equimolar quantities of both 3R-tau and 4Rtau. ${ }^{12}$ In one recent report both insoluble 3R-tau and 4R-tau were demonstrated in a subset of AGD brains. ${ }^{15}$

In addition to frank dementia which is reported in $12-50 \%$ of patients with a diagnosis of AGD, mild cognitive impairment, personality changes, and advancing age have also been associated with this condition. ${ }^{16-18}$ In demented individuals with concomitant Alzheimer's disease pathology, AGD is typically associated with milder Braak (I-III) staging and often lack other pathologies associated with dementia. ${ }^{18}$ The prevalence in unselected necropsy series of approximately $5 \%{ }^{18}$ is similar to results obtained from series of dementia cases. ${ }^{19}$ AGD is found in significantly higher proportions in neurodegenerative series, ranging from $33 \%$ to $40 \% .{ }^{17}$ Other neurodegenerative disorders associated with AGD include dementia with Lewy bodies (DLB) and MSA. Although rare cases of AGD combined with Parkinson's disease ${ }^{1820} 21$ and motor neurone disease ${ }^{17}$ have been encountered, the combination of the three pathologies in a single case has not yet been reported.
Although the conventional teaching is that neurodegenerative diseases are clinically and pathologically distinct, recent advances in molecular biology have blurred the boundaries between many of these disorders. The cooccurrence of tau and $\alpha$-synuclein inclusions is well recognised in MSA, DLB, combined Parkinson's disease and Alzheimer's disease, and the so called Lewy body variant of Alzheimer's disease. Pathological forms of both proteins have been recognised in typical Lewy bodies, ${ }^{6}$ in Guamanian Parkinsonism-dementia complex cases, ${ }^{22}$ and in members of the Contursi kindred who suffer from rare familial Parkinson's disease related to a missense mutation in the $\alpha$-synuclein gene. ${ }^{23}$ Moreover, recent studies suggest that in vitro tau fibrillisation is initiated by and dependent upon the presence of $\alpha$-synuclein. ${ }^{24}$

Dysfunction of the ubiquitin-proteasomal degradation system has increasingly been implicated in the process of neuronal degeneration and pathological protein aggregation. ${ }^{25}$ Both tau and synuclein fibrillisation occur as a reaction to oxidative stress or nitration. ${ }^{26}$ At a certain threshold, proteasomal function in susceptible cell populations-such as motor neurones or nigral dopaminergic neurones-may be overwhelmed, leading to decreased protein turnover and pathological aggregation. This idea is further supported by the finding that ubiquitinated and nitrated protein aggregates occur as a direct result of proteasomal inhibition. ${ }^{27}$ Whether or not the cellular inclusions are truly pathogenic or represent markers of neurodegeneration remains debatable. Nevertheless, the investigation of protein aggregates will certainly help to clarify mechanisms of neurodegeneration and may soon converge on a common pathogenic process. The co-occurrence of multiple neurodegenerative pathologies and inclusion types in our patient and other similar cases further suggest that these processes may share a common underlying aetiology.

\section{ACKNOWLEDGEMENTS}

We are indebted to the family members of the patient studied for making the research described here possible. This work was supported by grants from the National Institute on Aging of the National Institutes of Health (AG10124, AG09215). JED is supported by an advanced career development award from the Medical Research Service of the Department of Veteran Affairs. MSF is supported by a mentored clinical scientist development award from the National Institute on Aging (AG20073). JQT is the William Maul Measey-Truman G. Schnabel, Jr. Professor of Geriatric Medicine and Gerontology. AS is supported by a grant from the Agency for Healthcare Research and Quality (AHRQ No K-08 HS00004).

\section{Authors' affiliations}

T-W Liang, J E Duda, L McCluskey, A Siderowf, Department of Neurology, University of Pennsylvania School of Medicine, Philadelphia, Pennsylvania, USA

M S Forman, J Q Trojanowski, Department of Pathology and Laboratory Medicine, University of Pennsylvania School of Medicine

Competing interests: none declared

Correspondence to: Dr Tsao-Wei Liang, Parkinson's Disease Research, Education, and Clinical Center, Philadelphia VA Medical Center, University and Woodland Avenues, Philadelphia, PA 19104, USA; liangtw@mail.med.upenn.edu

Received 16 February 2004

In revised form 26 May 2004

Accepted 26 May 2004

\section{REFERENCES}

1 Rodgers-Johnson P, Garruto RM, Yanagihara R, et al. Amyotrophic lateral sclerosis and parkinsonism-dementia on Guam: a 30-year evaluation of clinical and neuropathologic trends. Neurology 1986;36:7-13. 
2 Konagaya M, Kato T, Sakai $M$, et al. A clinical and pathological study of a Japanese case of amyotrophic lateral sclerosis/parkinsonism-dementia complex with family history. J Neurol 2003;250:164-70.

3 Zoccolella S, Palagano G, Fraddosio A, et al. ALS-plus: 5 cases of concomitant amyotrophic lateral sclerosis and parkinsonism. Neurol Sci 2002;23(suppl 2):S123-4.

4 Judkins AR, Forman MS, Uryu K, et al. Co-occurrence of Parkinson's disease with progressive supranuclear palsy. Acta Neuropathol (Berl) 2002; 103:526-30.

5 Takanashi M, Ohta S, Matsuoka S, et al. Mixed multiple system atrophy and progressive supranuclear palsy: a clinical and pathological report of one case. Acta Neuropathol (Berl) 2002;103:82-7.

6 Testa D, Tiranti V, Girotti F. Unusual association of sporadic olivopontocerebellar atrophy and motor neuron disease. Neurol Sci 2002;23:243-5.

7 Hedera P, Lerner AJ, Castellani R, et al. Concurrence of Alzheimer's disease Parkinson's disease, diffuse Lewy body disease, and amyotrophic lateral sclerosis. J Neurol Sci 1995;128:219-24.

8 Greenberg SG, Davies P. A preparation of Alzheimer paired helical filaments that displays distinct tau proteins by polyacrylamide gel electrophoresis. Proc Natl Acad Sci USA 1990;87:5827-31.

9 Giasson BI, Duda JE, Murray IV, et al. Oxidative damage linked to neurodegeneration by selective alpha-synuclein nitration in synucleinopathy lesions. Science 2000;290:985-9.

10 Jellinger KA. Dementia with grains (argyrophilic grain disease). Brain Pathol 1998;8:377-86.

11 Braak H, Braak E. Argyrophilic grains: characteristic pathology of cerebral cortex in cases of adult onset dementia without Alzheimer changes. Neurosci Lett 1987:76:124-7.

12 Lee VM, Goedert M, Trojanowski JQ. Neurodegenerative tauopathies. Annu Rev Neurosci 2001;24:1121-59.

13 Ferrer I, Barrachina $M$, Tolnay $M$, et al. Phosphorylated protein kinases associated with neuronal and glial tau deposits in argyrophilic grain disease. Brain Pathol 2003;13:62-78.

14 Togo T, Sahara N, Yen SH, et al. Argyrophilic grain disease is a sporadic 4repeat tauopathy. J Neuropathol Exp Neurol 2002;61:547-56.
15 Zhukareva V, Shah K, Uryu K, et al. Biochemical analysis of tau proteins in argyrophilic grain disease, Alzheimer's disease, and Pick's disease: a comparative study. Am J Pathol 2002;161:1135-41.

16 Tolnay M, Spillantini MG, Goedert M, et al. Argyrophilic grain disease: widespread hyperphosphorylation of tau protein in limbic neurons. Acta Neuropathol (Berl) 1997;93:477-84.

17 Martinez-Lage P, Munoz DG. Prevalence and disease associations of argyrophilic grains of Braak. J Neuropathol Exp Neurol 1997;56:157-64.

18 Braak H, Braak E. Argyrophilic grain disease: frequency of occurrence in different age categories and neuropathological diagnostic criteria. I Neural Transm 1998; 105:801-19.

19 Togo T, Cookson N, Dickson DW. Argyrophilic grain disease: neuropathology, frequency in a dementia brain bank and lack of relationship with apolipoprotein E. Brain Pathol 2002;12:45-52.

20 Ikeda K, Akiyama H, Kondo H, et al. A study of dementia with argyrophilic grains. Possible cytoskeletal abnormality in dendrospinal portion of neurons and oligodendroglia. Acta Neuropathol (Berl) 1995;89:409-14.

21 Tolnay M, Schwietert M, Monsch AU, et al. Argyrophilic grain disease: distribution of grains in patients with and without dementia. Acta Neuropathol (Berl) 1997;94:353-8.

22 Forman MS, Schmidt ML, Kasturi S, et al. Tau and alpha-synuclein pathology in amygdala of Parkinsonism-dementia complex patients of Guam. Am J Pathol 2002; 160:1725-31.

23 Duda JE, Giasson $\mathrm{Bl}$, Mabon ME, et al. Concurrence of alpha-synuclein and tau brain pathology in the Contursi kindred. Acta Neuropathol (Berl) 2002;104:7-11.

24 Giasson BI, Forman MS, Higuchi $M$, et al. Initiation and synergistic fibrillization of tau and alpha-synuclein. Science 2003;300:636-40.

25 Giasson BI, Lee VM. Are ubiquitin pathways central to Parkinson's disease? Cell 2003;1 14:1-8.

26 Ischiropoulos $\mathbf{H}$, Beckman JS. Oxidative stress and nitration in neurodegeneration: cause, effect, or association? J Clin Invest 2003;111:163-9.

27 Hyun DH, Lee M, Halliwell B, et al. Proteasomal inhibition causes the formation of protein aggregates containing a wide range of proteins, including nitrated proteins. J Neurochem 2003;86:363-73. 